\title{
Pair Densities in a Two-dimensional Electron Gas (Jellium) at Strong Coupling from Scattering Theory with Kukkonen-Overhauser Effective Interactions
}

\author{
F. Capurro, R. Asgaria, B. Davoudi ${ }^{\text {a }}$, M. Polini, and M. P. Tosi \\ NEST-INFM and Classe di Scienze, Scuola Normale Superiore, I-56126 Pisa, Italy \\ a Also at Institute for Studies in Theoretical Physics and Mathematics, Tehran 19395-5531, Iran \\ Reprint requests to Prof. M. P. T.; Fax: +39-050-563513; E-mail: tosim@sns.it
}

Z. Naturforsch. 57 a, 237-243 (2002); received March 8, 2002

\begin{abstract}
We present a calculation of the spin-averaged and spin-resolved pair distribution functions for a homogeneous gas of electrons moving in a plane with $e^{2} / r$ interactions at coupling strength $r_{\mathrm{s}}=10$. The calculation is based on the solution of a two-electron scattering problem for both parallelspin- and antiparallel-spin-pairs interacting via effective spin-dependent many-body potentials. The scattering potentials are modeled within the approach proposed by Kukkonen and Overhauser to treat exchange and correlations under close constraints imposed by sum rules. We find very good agreement with quantum MonteCarlo data for the spin-averaged pair density. We also find that short-range pairing between parallel-spin electrons is beginning to emerge in the paramagnetic fluid at this coupling strength, as a precursor of a transition to a fully spin-polarized fluid state occurring at stronger coupling.
\end{abstract}

Key words: Electron Gas; Electron-electron Interactions; Exchange; Correlation;

Dielectric and Magnetic Response.

\section{Introduction}

Electronic fluids with essentially two-dimensional (2D) dynamics in semiconductor quantum wells present a very rich phenomenology, especially in the low-electron-density regime corresponding to strong coupling [1]. Many of the electron-electron interaction effects can be understood with the help of the homogeneous electron-gas (jellium) model [2]. A central role in the theory is played by the electron-pair distribution function $g(r)$ and by its spin-resolved components $g_{\uparrow \uparrow}(r)$ and $g_{\uparrow \downarrow}(r)$, which provide essential input in the construction of exchange and correlation energy functionals for applications of density functional theory (DFT) [3]. A number of recent developments in DFT [4 - 6] have also drawn attention to the utilization of the inhomogeneous electron-pair density, that is the diagonal part of the two-body reduced density matrix of the inhomogeneous electronic system [7].

It is well known that the value of $g(r)$ in jellium is determined in the limit $r \rightarrow 0$ by the scattering events between pairs of electrons with antiparallel spins, leading to an exact cusp condition which relates the logarithmic derivative of $g_{\uparrow \downarrow}(r)$ and $g(r)$ at contact to the Bohr radius $a_{\mathrm{B}}$ [8]. The value of $g_{\uparrow \uparrow}(r)$ vanishes in the same limit as a consequence of the Pauli exclusion principle. Early calculations of $g_{\uparrow \downarrow}(0)$ by many-body techniques exploited the dominant role of the electron-electron ladder diagrams in determining this quantity in both $3 \mathrm{D}$ jellium $[9,10]$ and $2 \mathrm{D}$ jellium [11, 12]. In a seminal work Overhauser [13] developed a wholly different approach to the evaluation of $g_{\uparrow \downarrow}(0)$ in 3D jellium, in which he tackled the scattering problem through the solution of an effective Schrödinger equation for the relative motion of the electronic pair. The approach of Overhauser was later used to evaluate $g_{\uparrow \downarrow}(0)$ in 2D jellium [14] and was extended by Gori-Giorgi and Perdew [15] to calculate $g(r)$ at finite $r$ in 3D jellium by means of an accurate numerical solution of Overhauser's twobody Schrödinger equation. This equation involves a simple model for the scattering potential, which is built as the potential of an electron at the centre of a Wigner-Seitz sphere of uniformly distributed positive charge. 
Comparisons with quantum MonteCarlo (QMC) data show that the above extension of Overhauser's approach for the evaluation of pair densities away from contact in 3D jellium gives quite good results in the short-range part of $g(r)$, where two-body collisions may still be expected to be most important [15]. In a full calculation of pair densities by this approach, however, one would be trying to reduce a many-electron problem to a two-electron scattering problem and should therefore give careful attention to a proper inclusion of many-body effects into the scattering potential entering the effective two-body Schrödinger equation. In this perspective Davoudi et al. [16] have adopted a self-consistent Hartree scheme for the determination of the effective scattering potential, selfconsistency being allowed by the fact that the Hartree potential generated by an "average" electron is determined by the density of electrons around the same electron and this is in turn described by $g(r)$. It was shown in the above study that such a self-consistent Hartree approach yields a very good account of $g(r)$ in $3 \mathrm{D}$ jellium over the whole range of interelectron distances in a range of coupling strengths $r_{\mathrm{s}}$ extending up to at least $r_{\mathrm{s}}=10$. Rather surprisingly, the Hartree approximation also gives a quite reasonable, though not equally accurate, account of the spin-resolved pair densities over the same range of coupling strengths.

It was also shown in the same study by Davoudi et al. [16] that the Hartree approximation has more restricted usefulness for the calculation of the pair densities in 2D jellium. Indeed, while it gives in this case a very good account of QMC data at $r_{\mathrm{s}}=1$, quantitative discrepancies between theoretical results and the available QMC data become evident already at $r_{\mathrm{s}}=5$. It is a general fact that the role of exchange and correlations becomes more important with decreasing dimensionality of the many-electron fluid, and this is directly evidenced in the QMC data by the emergence of an incipient first-neighbour shell in 2D jellium at $r_{\mathrm{s}}=5$ [17]. This feature is not reproduced by the Hartree approximation for the scattering potential entering the two-body Schrödinger equation [16].

In the present work we examine whether the inclusion of exchange and correlation in the effective two-body scattering problem may usefully allow one to transcend the results yielded by the Hartree approximation at strong coupling. We do so by recourse to the electron-electron effective interactions derived by Kukkonen and Overhauser [18] and focus on 2D jellium in the strong-coupling regime corresponding to $r_{\mathrm{s}}=10$, where QMC data by Rapisarda and Senatore [19] allow a quantitative test of our results for $g(r)$. We also examine the behaviour of the spin-resolved pair densities, in relation to the first-order transition from the paramagnetic to the fully spin-polarized state that has been reported to occur in 2D jellium at $r_{\mathrm{s}} \approx 20$ from QMC studies [20]. In our present formulation the effective scattering potentials acquire a dependence on the relative orientation of the spins of the electron pair, but are not self-consistently dependent on the spin-resolved pair densities.

The paper is organized as follows. Section 2 gives the essential theoretical background, and Sect. 3 reports and discusses our numerical results. A brief summary concludes the paper in Section 4.

\section{Essential Theory}

We consider the 2D jellium model at average electron density $n$ and define its coupling strength $r_{\mathrm{s}}$ through the relation $\pi\left(r_{\mathrm{s}} a_{\mathrm{B}}\right)^{2}=1 / n$. Let $\Psi_{\boldsymbol{k}, \boldsymbol{K}}^{\sigma \sigma^{\prime}}(\boldsymbol{r}, \boldsymbol{R})$ be the solution of the two-electron scattering problem in some spin-dependent effective potential $V_{\sigma \sigma^{\prime}}(r)$. Here, $\boldsymbol{r}=\boldsymbol{r}_{1}-\boldsymbol{r}_{2}$ and $\boldsymbol{R}=\left(\boldsymbol{r}_{1}+\boldsymbol{r}_{2}\right) / 2$ are the relative and centre-of-mass coordinates of the electron pair, while the conjugate momenta are $\boldsymbol{k}$ and $\boldsymbol{K}$ and the spin indices of the pair are $\sigma$ and $\sigma^{\prime}$. The distribution functions $g_{\sigma \sigma^{\prime}}(r)$ are to be obtained as

$$
g_{\sigma \sigma^{\prime}}(r)=\left\langle\left\langle\left|\Psi_{k, \boldsymbol{K}}^{\sigma \sigma^{\prime}}(\boldsymbol{r}, \boldsymbol{R})\right|^{2}\right\rangle_{\theta}\right\rangle_{p(k)}
$$

where $\left\langle\langle\cdots\rangle_{\theta}\right\rangle_{p(k)}$ represents averages over the scattering angle $\theta$ and over the probability $p(k)$ of finding two electrons with relative momentum $k$ in the 2D electron gas,

$$
\left\langle\langle\mathcal{O}\rangle_{\theta}\right\rangle_{p(k)} \equiv \frac{1}{2 \pi} \int_{0}^{k_{\mathrm{F}}} \mathrm{d} k p(k) \int \mathrm{d} \Omega \mathcal{O} .
$$

The function $p(k)$ can be calculated from the momentum distribution $n(k)$, the result being

$$
p(k)=\frac{16 k}{\pi k_{\mathrm{F}}^{2}}\left[\arccos \left(\frac{k}{k_{\mathrm{F}}}\right)-\frac{k}{k_{\mathrm{F}}} \sqrt{1-\frac{k^{2}}{k_{\mathrm{F}}^{2}}}\right],
$$

when one uses the momentum distribution of the ideal 2D Fermi gas with Fermi wave number $k_{\mathrm{F}}=$ $\sqrt{2} /\left(r_{\mathrm{s}} a_{\mathrm{B}}\right)$ [21]. The charge-charge and spin-spin correlations in the paramagnetic fluid are given by $g(r)=$ 
$\left[g_{\uparrow \uparrow}(r)+g_{\uparrow \downarrow}(r)\right] / 2$ and $g_{\mathrm{d}}(r)=\left[g_{\uparrow \uparrow}(r)-g_{\uparrow \downarrow}(r)\right] / 2$, respectively.

The pair wave functions can be written in the form of angular-momentum expansions [15], leading to the results

$$
g_{\uparrow \uparrow}(r)=\frac{4}{r} \sum_{\ell=1 \text { (odd) }}^{\infty}\left\langle\left|\Phi_{\ell, k}^{(\uparrow \uparrow}(r)\right|^{2}\right\rangle_{p(k)}
$$

and

$$
\begin{aligned}
g_{\uparrow \downarrow}(r)=\frac{1}{r}\left[\left\langle\left|\Phi_{0, k}^{(\uparrow \downarrow)}(r)\right|^{2}\right\rangle_{p(k)}\right. & \\
& \left.+2 \sum_{\ell=1}^{\infty}\left\langle\left|\Phi_{\ell, k}^{(\uparrow \downarrow)}(r)\right|^{2}\right\rangle_{p(k)}\right],
\end{aligned}
$$

where the wave functions $\Phi_{\ell, k}^{\left(\sigma \sigma^{\prime}\right)}(r)$ obey the Schrödinger equation

$$
\begin{gathered}
{\left[-\frac{\hbar^{2}}{2 \mu} \frac{\mathrm{d}^{2}}{\mathrm{~d} r^{2}}+\frac{1}{2 \mu r^{2}}\left(\boldsymbol{L}^{2}-\frac{1}{4} \hbar^{2}\right)+V_{\sigma \sigma^{\prime}}(r)\right] \Phi_{\ell, k}^{\left(\sigma \sigma^{\prime}\right)}(r)} \\
=\frac{\hbar^{2} k^{2}}{2 \mu} \Phi_{\ell, k}^{\left(\sigma \sigma^{\prime}\right)}(r) .
\end{gathered}
$$

Here, $\mu=m / 2$ is the reduced electron mass and $\boldsymbol{L}^{2}=\hbar^{2} \ell^{2}$ with $\ell=0, \pm 1, \pm 2, \cdots$.

The form of (4) ensures that the relation $g_{\uparrow \uparrow}(0)=0$ is satisfied, since all functions $\Phi_{\ell, k}^{\left(\sigma \sigma^{\prime}\right)}(r)$ vanish at the origin for $\ell \neq 0$. The cusp condition is $\mathrm{d} \ln g_{\uparrow \downarrow}(r) /\left.\mathrm{d} r\right|_{r=0}=2 / a_{\mathrm{B}}$ in $2 \mathrm{D}$ and is satisfied if $V_{\uparrow \downarrow}(r)$ tends to the bare Coulomb potential for $r \rightarrow 0$ [16]. It is also easily seen that the HartreeFock results for $g_{\uparrow \uparrow}(r)$ and $g_{\uparrow \downarrow}(r)$ are recovered if $V_{\sigma \sigma^{\prime}}(r)$ is set to zero in (6) [15]. Finally, the normalization of the wave functions and of the probability function $p(k)$ ensures the charge neutrality condition, $n \int \mathrm{d} r[g(r)-1]=-1$.

In the following we adopt for the scattering potentials $V_{\sigma \sigma^{\prime}}(r)$ the form obtained by Kukkonen and Overhauser [18], which includes exchange and correlations through a linear-response treatment of the polarization induced by an "average" electron of given spin in the surrounding electron gas and tends to the bare Coulomb potential $e^{2} / r$ for $r \rightarrow 0$. In Fourier transform their result is

$$
\begin{array}{r}
\tilde{V}_{\sigma \sigma^{\prime}}(q)=v_{q}\left\{\frac{1+\left[1-G_{+}(q)\right] G_{+}(q) Q(q)}{1+\left[1-G_{+}(q)\right] Q(q)}\right. \\
\left.-\sigma \sigma^{\prime} \frac{G_{-}^{2}(q) Q(q)}{1-G_{-}(q) Q(q)}\right\},
\end{array}
$$

where, by convention, the product $\sigma \sigma^{\prime}$ equals +1 for parallel spins and -1 for antiparallel spins. In (7) $v_{q}=2 \pi e^{2} / q$ is the Fourier transform of the Coulomb potential, $Q(q)=v_{q} \chi_{0}(q)$ with $\chi_{0}(q)$ the static density response function of the ideal 2D Fermi gas as first calculated by Stern [22], and $G_{ \pm}(q)$ are the local field factors accounting for exchange and correlations in the dielectric and spin response of the electron gas [2]. As discussed further below, the local field factors are available from QMC data [23 - 25] extending in 2D up to $r_{\mathrm{s}}=10$.

We conclude this section by recalling how exact sum rules and limiting behaviours enter the scattering potentials $\tilde{V}_{\sigma \sigma^{\prime}}(q)$ at both small and large momentum transfers [2]. The latter behaviours are determined by the values of $g(0)$ and of the correlation kinetic energy (see Appendix). The compressibility and spin susceptibility sum rules, on the other hand, determine the limiting behaviours of the local field factors at low momenta according to the relations

$$
\lim _{q \rightarrow 0} G_{ \pm}(q)=A_{ \pm} \frac{q}{k_{\mathrm{F}}}
$$

where

$$
A_{+}=\frac{1}{\sqrt{2} r_{\mathrm{s}}}\left(1-\frac{\kappa_{0}}{\kappa}\right)
$$

and

$$
A_{-}=\frac{1}{\sqrt{2} r_{\mathrm{S}}}\left(1-\frac{\chi_{\mathrm{P}}}{\chi_{\mathrm{s}}}\right)
$$

Here, $\kappa$ and $\chi_{\mathrm{s}}$ are the compressibility and the spin susceptibility of $2 \mathrm{D}$ jellium, with $\kappa_{0}$ and $\chi_{\mathrm{P}}$ being the corresponding values for the ideal 2D Fermi gas. Using also the result [22]

$$
\lim _{q \rightarrow 0} \chi_{0}(q)=\frac{m}{\pi \hbar^{2}}
$$

one easily finds

$$
\lim _{q \rightarrow 0} \tilde{V}_{\sigma \sigma^{\prime}}=\frac{\pi \hbar^{2}}{m}\left[\left(2-\frac{\kappa_{0}}{\kappa}\right)-\sigma \sigma^{\prime} \frac{\chi_{\mathrm{s}}}{\chi_{\mathrm{P}}}\left(1-\frac{\chi_{\mathrm{P}}}{\chi_{\mathrm{s}}}\right)^{2}\right]
$$

Notice that for sufficiently large values of $\chi_{\mathrm{s}} / \chi_{\mathrm{P}}$ the spin term in (12) leads to an effective attraction between parallel-spin electrons at low momenta and to a corresponding effective repulsion between antiparallel-spin electrons. This fact favours the formation of fluctuating domains of spin magnetization 


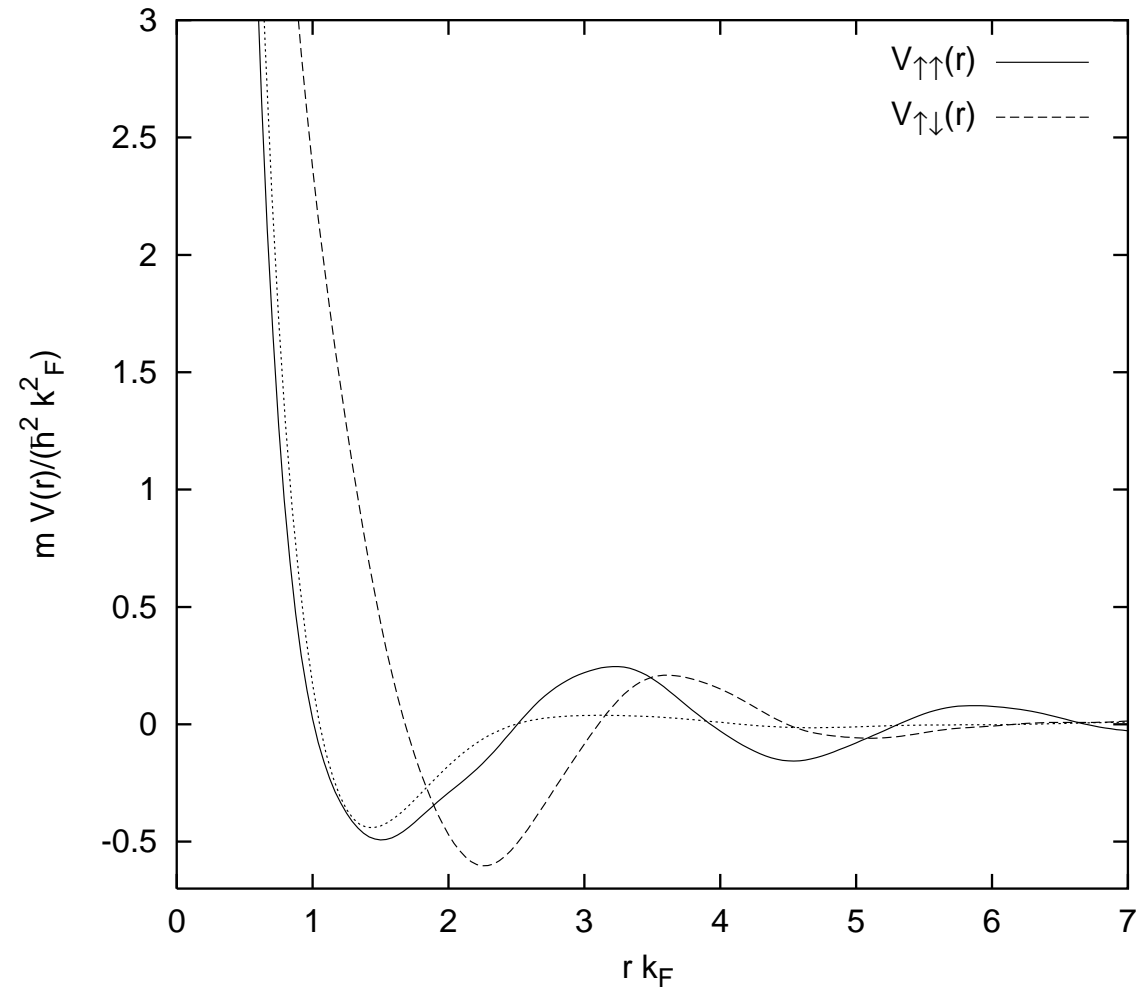

Fig. 1. Effective electron-electron scattering potentials in the two-dimensional electron gas at $r_{\mathrm{s}}=10$ (in units of $\left.\hbar^{2} k_{\mathrm{F}}^{2} / m\right)$, as functions of $r k_{\mathrm{F}}$. Full line: $V_{\uparrow \uparrow}(r)$; dashed line: $V_{\uparrow \downarrow}(r)$. The dotted line shows the result of the self-consistent Hartree approximation, from [16]. in the paramagnetic electron fluid as its coupling strength parameter is raised towards the transition to a fully spin-polarized state [26].

\section{Numerical Results}

The numerical solution of (6) requires as input the local field factors entering the scattering potentials in (7). These are available in analytical form from QMC data in 2D jellium up to $r_{\mathrm{s}}=10$ [27]. It turns out that the solution of the scattering problem is quite sensitive to the details of this input, so that we thought it advisable to simplify the form of the fit of the QMC data at long wavelengths. The details of the new fit and its results, that we have used in the calculations reported immediately below, are presented in the Appendix.

Figures 1 and 2 report the scattering potentials in 2D jellium at $r_{\mathrm{s}}=10$ as functions of the distance $r$ and in Fourier transform, respectively. The comparison with the spin-independent potential obtained in the Hartree approximation, which is given in Fig. 1, shows that the inclusion of exchange and correlation gives rise to oscillations extending quite far away from the electron at the origin. In addition, the minima in the two scattering potentials are well separated from each other, with that for parallel-spin electrons showing at this value of $r_{\mathrm{s}}$ a marked minimum at short distance in approximate correspondence with the main minimum in the Hartree-model potential. It can be seen from Fig. 2 that these features in $r$ space correspond in Fourier transform to a decrease in the effective repulsion between parallel-spin electrons over a wide region of scattering momenta extending up to $2 k_{\mathrm{F}}$, while antiparallel-spin electrons feel an enhanced repulsion in the same region of momenta. As was discussed in [26], the emergence of effective interactions favouring spin alignments over domains on a scale of distance which is becoming of the order of $\left(2 k_{\mathrm{F}}\right)^{-1}$ is signalling the approach of the paramagnetic fluid to a transition into a spin-ordered phase having a space-independent order parameter.

The above statement is demonstrated in the results that we obtain for the "difference" spin distribution function $g_{\mathrm{d}}(r)=\left[g_{\uparrow \uparrow}(r)-g_{\uparrow \downarrow}(r)\right] / 2$ at $r_{\mathrm{s}}=10$, which are reported in Figure 3. This shows that $g_{\mathrm{d}}(r)$ is slightly positive at short distance, corresponding to a 

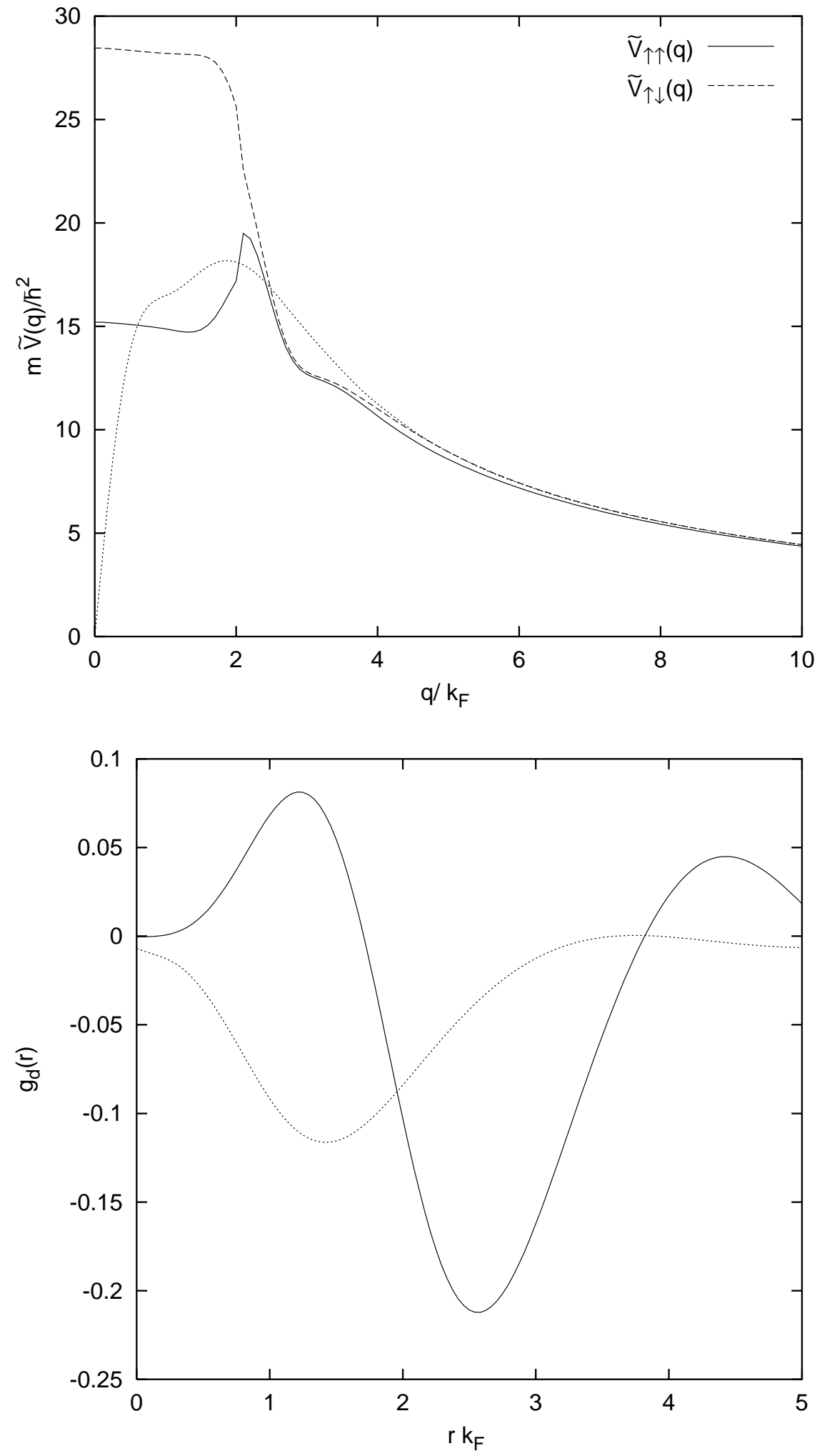

Fig. 2. Effective electronelectron scattering potentials in the two-dimensional electron gas at $r_{\mathrm{s}}=10$ (in units of $\left.\hbar^{2} / m\right)$, as functions of $q / k_{\mathrm{F}}$. Full line: $\tilde{V}_{\uparrow \uparrow}(q)$; dashed line: $\tilde{V}_{\uparrow \downarrow}(q)$. The dotted line shows the result of the selfconsistent Hartree approximation, from [16].

Fig. 3. The "difference" pair distribution function $g_{\mathrm{d}}(r)=$ $\left[g_{\uparrow \uparrow}(r)-g_{\uparrow \downarrow}(r)\right] / 2$ in the twodimensional electron gas at $r_{\mathrm{s}}=10$, as a function of $r k_{\mathrm{F}}$. Full line: from KukkonenOverhauser scattering potentials. The dotted line shows the result of the self-consistent Hartree approximation, from [16]. 


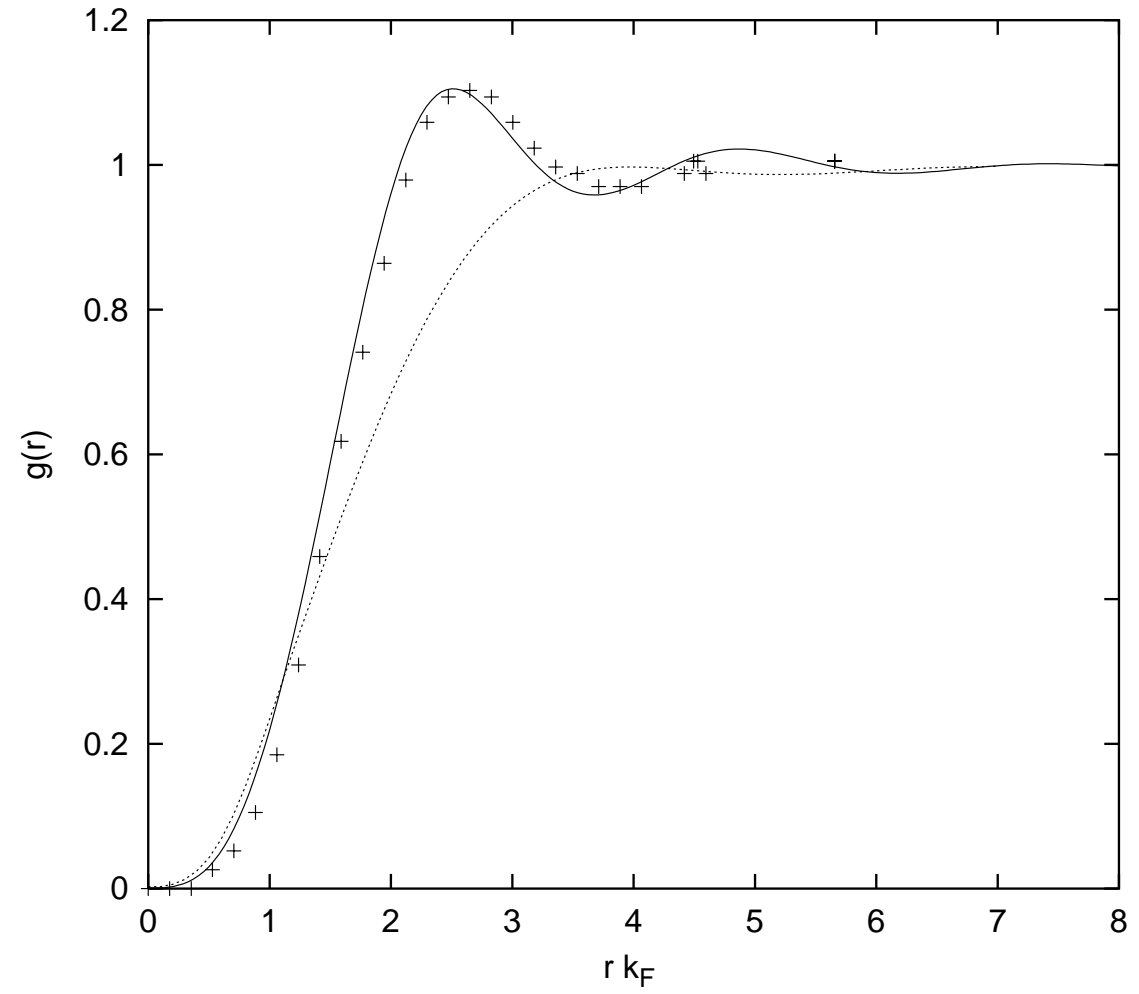

Fig. 4. The spin-averaged pair distribution function $g(r)=$ $\left[g_{\uparrow \uparrow}(r)+g_{\uparrow \downarrow}(r)\right] / 2$ in the twodimensional electron gas at $r_{\mathrm{s}}=10$, as a function of $r k_{\mathrm{F}}$. Full line: from KukkonenOverhauser scattering potentials. The dotted line shows the result of the self-consistent Hartree approximation, from [16]. The crosses are from QMC results in [19]. slight tendency for parallel-spin electrons to be first neighbours in the paramagnetic fluid. On the other hand the Hartree approximation, the result of which is also shown in Fig. 3, still gives negative values for $g_{\mathrm{d}}(r)$ in the same region of space, corresponding to the preferred short-range pairing between antiparallel spins which is characteristic of the fluid in the deep paramagnetic phase. We should point out that, while the present method to include exchange and correlation thus appears to be qualitatively useful in regard to the spin-resolved distribution functions, its results are quite sensitive to the detailed shape of $G_{-}(q)$, and their quantitative validity cannot be tested against QMC data.

Finally, Fig. 4 reports the spin-averaged pair distribution function $g(r)$ in 2D jellium at $r_{\mathrm{s}}=10$, in comparison with the QMC data of Rapisarda and Senatore [19] and with those obtained within the Hartree approximation. It is evident that the present approach yields a fully quantitative account of the QMC data, and in particular reproduces the presence of a firstneighbour shell at this value of the coupling strength which is instead completely missed in the Hartree approximation to the scattering potentials.

\section{Summary}

In summary, we have shown that the inclusion of exchange and correlation in the effective many-body potentials governing the scattering between electron pairs in the two-dimensional electron gas at strong coupling provides a quantitative account of the data on the spin-averaged pair distribution function. It also yields apparently reasonable results for the difference in the distribution functions bertween parallel- and antiparallel-spin electron pairs on the approach to magnetic ordering in the electron fluid.

Regretfully, no data are as yet available at stronger couplings closer to the magnetic ordering transition, which would allow further quantitative tests of the theory. At a coupling strength corresponding to $r_{\mathrm{s}}=5$, on the other hand, the available QMC data indicate that the present determination of the scattering potentials does not have a similar quantitative usefulness. This is somewhat surprising, since in their derivation the Kukkonen-Overhauser potentials involve a linear-response assumption. The inclusion of selfconsistency in the theory may therefore be expected to be important. 


\section{Acknowledgements}

This work was partially suported by MIUR under the PRIN-2001 Programme.

\section{Appendix. Local Field Factors from QMC Data on the 2D Electron Gas}

In this work we have redetermined the local field factors from QMC data by the same procedure that we have used in our previous work [27], using simpler expressions for their functional form with the aim of reducing the sensitivity of the results to small fluctuations in the input data. The form that we have taken at $r_{\mathrm{s}}=5$ and $r_{\mathrm{s}}=10$ is

$$
\begin{aligned}
G_{ \pm}(x)= & \frac{A_{ \pm} x+\left(B_{ \pm}+C_{ \pm} x\right)(x / 2)^{8}}{1+(x / 2)^{8}} \\
& +\left(a_{ \pm} x^{b_{ \pm}}+c_{ \pm} x^{d_{ \pm}}\right) \exp \left[-\left(x-e_{ \pm}\right)^{2} / f_{ \pm}\right]
\end{aligned}
$$

[1] E. Abrahams, S. V. Kravchenko, and M. P. Sarachik, Rev. Mod. Phys. 73, 251 (2001).

[2] For a recent review of many-body aspects of electron gas theory see M. P. Tosi, in "Electron Correlations in the Solid State", ed. N. H. March, Imperial College Press, London 1999, p. 5.

[3] See, e. g. R. M. Dreizler and E. K. U. Gross, "Density Functional Theory, an Approach to the Quantum Many-Body Problem", Springer, Berlin 1990.

[4] A. Gonis, T. C. Schulthess, J. van Ek, and P. E. A. Turchi, Phys. Rev. Lett. 77, 2981 (1996).

[5] P. Ziesche, in "Electron Correlations and Material Properties", ed. A. Gonis, N. Kioussis, and M. Ciftan, Kluwer, New York 1999, p. 361.

[6] P. Ziesche, J. Chem. Phys. 115, 9110 (2001).

[7] E. R. Davidson, "Reduced Density Matrices in Quantum Chemistry", Academic, New York 1976.

[8] J. C. Kimball, Phys. Rev. A 7, 1648 (1973).

[9] H. Yasuhara, Solid State Commun. 11, 1481 (1972).

[10] B. B. J. Hede and J. P. Carbotte, Can. J. Phys. 50, 1756 (1972).

[11] D. L. Freeman, J. Phys. C 16, 711 (1983).

[12] S. Nagano, K. S. Singwi, and S. Ohnishi, Phys. Rev. B 29, 1209 (1984).

[13] A. W. Overhauser, Can. J. Phys. 73, 683 (1995).

[14] M. Polini, G. Sica, B. Davoudi, and M. P. Tosi, J. Phys.: Condens. Matter 13, 3591 (2001).
Table 1. Parameters of the local field factors in $2 \mathrm{D}$ jellium ${ }^{\mathrm{a}}$.

\begin{tabular}{rrrrrrr}
\hline$r_{\mathrm{s}}$ & \multicolumn{1}{c}{$a_{ \pm}$} & \multicolumn{1}{c}{$b_{ \pm}$} & \multicolumn{1}{c}{$c_{ \pm}$} & \multicolumn{1}{c}{$d_{ \pm}$} & \multicolumn{1}{c}{$e_{ \pm}$} & $f_{ \pm}$ \\
\hline 5 & 0.8198 & 12.7166 & -1.8773 & 11.6978 & -3.3076 & 2.8223 \\
& -1.6396 & 8.9557 & 2.8019 & 7.9622 & -5.8743 & 6.8942 \\
10 & 1.3063 & 10.3891 & -1.3087 & 10.3869 & 0.3794 & 1.1095 \\
& -2.1138 & 13.9579 & 2.1112 & 13.9582 & -2.3053 & 2.4713 \\
\hline
\end{tabular}

${ }^{\text {a }}$ For each value of $r_{\mathrm{s}}$ the first (second) row reports the values of the parameters for $G_{+}\left(G_{-}\right)$.

where $x=q / k_{\mathrm{F}}$. In (A.1) the quantities $A_{ \pm}$determine the behaviour of the local field factors in the long-wavelength limit (see (9) and (10)), while the quantities $B_{ \pm}$and $C_{ \pm}$govern the large- $q$ behaviours as determined by the radial distribution function at contact and by the true kinetic energy of the electron gas (see the discussion and the results given in [27]).

The other parameters in (A.1) serve instead to reproduce the QMC data on the local field factors in the intermediate range of wave number. Their numerical values are reported in Table 1.

[15] P. Gori-Giorgi and J. P. Perdew, Phys. Rev. B 64, 155102 (2001).

[16] B. Davoudi, M. Polini, R. Asgari, and M. P. Tosi, cond-mat/0201423.

[17] B. Tanatar and D. M. Ceperley, Phys. Rev. B 39, 5005 (1989).

[18] C. A. Kukkonen and A. W. Overhauser, Phys. Rev. B 20, 550 (1979).

[19] F. Rapisarda and G. Senatore, Austr. J. Phys. 49, 161 (1996).

[20] D. Varsano, S. Moroni, and G. Senatore, Europhys. Lett. 53, 348 (2001).

[21] P. Ziesche, J. Tao, M. Seidl, and J. P. Perdew, Int. J. Quantum Chem. 77, 819 (2000).

[22] F. Stern, Phys. Rev. Lett. 18, 546 (1967).

[23] S. Moroni, D. M. Ceperley, and G. Senatore, Phys. Rev. Lett. 69, 1837 (1992).

[24] G. Senatore, S. Moroni, and D. M. Ceperley, in "Quantum Monte Carlo Methods in Physics and Chemistry", ed. M. P. Nightingale and C. J. Umrigar, Kluwer, Dordrecht 1999.

[25] G. Senatore, S. Moroni, and D. Varsano, Solid State Commun. 119, 335 (2001).

[26] B. Davoudi, M. Polini, G. Sica, and M. P. Tosi, Solid State Commun. 121, 295 (2002).

[27] B. Davoudi, M. Polini, G. Giuliani, and M. P. Tosi, Phys. Rev. B 64, 153101 and 233110 (2001). 